\title{
Spatial Solitons and Induced Kerr Effects in Quasi-Phase-Matched Quadratic Media
}

\author{
Clausen, Carl A. Balslev; Bang, Ole; Kivshar, Yu.S.
}

Published in:

Physical Review Letters

Link to article, DOI:

10.1103/PhysRevLett.78.4749

Publication date:

1997

Document Version

Publisher's PDF, also known as Version of record

Link back to DTU Orbit

Citation (APA):

Clausen, C. A. B., Bang, O., \& Kivshar, Y. S. (1997). Spatial Solitons and Induced Kerr Effects in Quasi-PhaseMatched Quadratic Media. Physical Review Letters, 78(25), 4749-4752.

https://doi.org/10.1103/PhysRevLett.78.4749

\section{General rights}

Copyright and moral rights for the publications made accessible in the public portal are retained by the authors and/or other copyright owners and it is a condition of accessing publications that users recognise and abide by the legal requirements associated with these rights.

- Users may download and print one copy of any publication from the public portal for the purpose of private study or research.

- You may not further distribute the material or use it for any profit-making activity or commercial gain

- You may freely distribute the URL identifying the publication in the public portal

If you believe that this document breaches copyright please contact us providing details, and we will remove access to the work immediately and investigate your claim. 


\title{
Spatial Solitons and Induced Kerr Effects in Quasi-Phase-Matched Quadratic Media
}

\author{
Carl Balslev Clausen, ${ }^{1,2}$ Ole Bang, ${ }^{2}$ and Yuri S. Kivshar ${ }^{2}$ \\ ${ }^{1}$ Department of Mathematical Modelling, Technical University of Denmark, DK-2800 Lyngby, Denmark \\ ${ }^{2}$ Australian Photonics Cooperative Research Center, Research School of Physical Sciences and Engineering, \\ Optical Sciences Center, Australian National University, Canberra 0200 ACT, Australia
}

(Received 18 February 1997)

\begin{abstract}
We show that the evolution of the average intensity of $\mathrm{cw}$ beams in a quasi-phase-matched quadratic (or $\chi^{(2)}$ ) medium is strongly influenced by induced Kerr effects, such as self- and cross-phase modulation. We prove the existence of rapidly oscillating solitary waves (a spatial analog of the guidedcenter soliton) supported by the quadratic and induced cubic nonlinearities. [S0031-9007(97)03436-4]
\end{abstract}

PACS numbers: 42.65.Tg, 03.40.Kf, 42.65.Jx, 42.65.Ky

One of the most spectacular manifestations of nonlinearity in physical systems is the existence of solitary waves, which occur when dispersion (or diffraction) is balanced by nonlinearity. In nonlinear optics the fundamental equation to describe solitary waves is the nonlinear Schrödinger equation, valid for both pulse (temporal) and beam (spatial) propagation in a medium with cubic (or Kerr) nonlinearity. However, as has been already established (see Ref. [1] to cite a few), solitary waves can also exist in media with quadratic nonlinearity as a result of cascading, under the condition of phase matching.

Solitary waves in quadratic (or $\chi^{(2)}$ ) materials have attracted growing attention, because of the possibility to employ large second-order nonlinearities for the needs of all-optical switching. Such spatial solitary waves have been recently observed experimentally in a potassiumtitaynl-posphate bulk crystal and in a $\mathrm{LiNbO}_{3}$ slab waveguide [2]. However, the efficiency of the cascaded nonlinearities in those experiments was quite low, and thus high input powers were required. This was partly because of the limitations imposed by the use of conventional phase-matching techniques based on birefringence and temperature tuning.

In the context of second-harmonic generation the quasiphase-matching (QPM) technique is known as an attractive way to obtain good phase matching, and has been studied intensively (see Ref. [3] for a review). The QPM technique relies on the periodic modulation of the nonlinear susceptibility and/or refractive index, by which an additional (grating) wave vector is introduced, which can compensate for the mismatch between the wave vectors of the fundamental and second-harmonic waves. With the QPM technique, phase matching becomes possible at ambient temperatures, and does not introduce spatial walk off; the polarization with the largest nonlinearity can be used, and materials with strong nonlinearities can be explored, which are not phase matchable by angle or temperature tuning. The physics of QPM has been known since 1962 [4], but only recently have the experimental difficulties been overcome and stable techniques been developed, such as domain inversion in ferroelectric materi- als [5], proton exchange [6], and etching and cladding [7], to mention a few.

In view of this an important question is: Can $Q P M$ be employed to achieve self-trapping of light and to support spatial solitary waves in quadratic materials? The answer is not obvious, because resonances between the domain length of the periodic structure and the beam characteristic length might induce instability. In this Letter we therefore investigate self-trapping and propagation of spatial solitary waves in QPM quadratic media, and derive effective average equations that are shown to include both quadratic and periodicity-induced cubic nonlinearities. We prove the existence of a novel class of solitary waves, QPM solitons, supported by the competing nonlinearities, and we analyze their structure and stability.

We consider the interaction of a $\mathrm{cw}$ beam with the fundamental frequency $\omega$ and its second harmonic $(2 \omega)$, propagating in a QPM $\chi^{(2)}$ slab waveguide, where only the nonlinear susceptibility is modulated. Assuming nonlinearity to be of the same order as diffraction, the evolution of slowly varying beam envelopes is governed by the normalized equations (see, e.g., Refs. [3,4,8,9])

$$
\begin{aligned}
i \frac{\partial W}{\partial z}+\frac{1}{2} \frac{\partial^{2} W}{\partial x^{2}}+d(z) W^{*} V e^{-i \beta z} & =0, \\
i \frac{\partial V}{\partial z}+\frac{1}{4} \frac{\partial^{2} V}{\partial x^{2}}+d(z) W^{2} e^{i \beta z} & =0,
\end{aligned}
$$

where $W(x, z)$ and $V(x, z)$ are the envelopes of the fundamental and the second harmonic, respectively. The parameter $\beta=\Delta k\left|k_{\omega}\right| x_{0}^{2}$ is proportional to the phase mismatch $\Delta k=2 k_{\omega}-k_{2 \omega}, k_{\omega}$ and $k_{2 \omega}$ being the wave numbers at the two frequencies. The normalization parameter $x_{0}$ is equal to the input beam width. Spatial walk off is neglected; it will usually not be present in QPM materials, since perpendicular or parallel polarization states can be employed. The transverse coordinate $x$ is measured in units of $x_{0}$, and the propagation coordinate $z$ is measured in units of the diffraction length $l_{d}=x_{0}^{2}\left|k_{\omega}\right|$. The spatial, periodic modulation of the nonlinear susceptibility $\chi^{(2)}$ is described by the QPM-grating function $d(z)$, 
whose amplitude is normalized to 1 , and whose domain length we define as $\pi / \kappa$. In general, the periodic function $d(z)$ can be expanded in a Fourier series

$$
d(z)=\sum_{n} d_{n} e^{i n \kappa z}
$$

where the summation is over all $n$ from $-\infty$ to $\infty$. In many physical applications the QPM grating can be well approximated by the square function depicted in Fig. 1, for which the Fourier series (2) contain only odd harmonics, $d_{2 n}=0$ and $d_{2 n+1}=2 / i \pi(2 n+1)$.

Inserting $d(z)$, given by Eq. (2), into Eqs. (1) and making the transformation $W(x, z)=w(x, z)$ and $V(x, z)=$ $v(x, z) \exp (i \tilde{\beta} z)$, we obtain the equations

$$
\begin{array}{r}
i \frac{\partial w}{\partial z}+\frac{1}{2} \frac{\partial^{2} w}{\partial x^{2}}+d_{m} w^{*} v+w^{*} v \sum_{n \neq m} d_{n} e^{i(n-m) \kappa z}=0, \\
i \frac{\partial v}{\partial z}+\frac{1}{4} \frac{\partial^{2} v}{\partial x^{2}}-\tilde{\beta} v+d_{-m} w^{2}+w^{2} \sum_{n \neq-m} d_{n} e^{i(n+m) \kappa z}=0,
\end{array}
$$

where $\tilde{\beta}=\beta-m \kappa$ is the effective phase-mismatch parameter for QPM of the $m$ th order. We assume that the QPM period is well controlled, so that $\beta \approx m \kappa$. This means that $\tilde{\beta}$ is of the order of one or less (ideally 0 ), even though $\beta$ might be large itself.

Equations (3) include coefficients that are periodically varying with the period $2 \pi / \kappa$. If $\kappa$ is sufficiently large, the dynamics could therefore be adequately described by averaged equations. Physically, $m \kappa \approx \beta \gg 1$ means that the coherence length $l_{c}=2 \pi / \Delta k$ is much smaller than the diffraction length $l_{d}$, since $\beta=2 \pi l_{d} / l_{c}$. To derive these equations we use an approach based on the asymptotic expansion technique, which has been successfully applied in many types of soliton problems [10].

We consider therefore the case where $\kappa \gg 1$ and expand the functions $w(x, z)$ and $v(x, z)$ in a Fourier series

$$
w=\sum_{n} w_{n} e^{i n m \kappa z}, \quad v=\sum_{n} v_{n} e^{i n m \kappa z},
$$

where $w_{n}(x, z)$ and $v_{n}(x, z)$ are assumed to vary slowly compared with $\exp (i \kappa z)$. This gives the equations for the coefficients $w_{n}$ and $v_{n}$. Now, following the reasoning of

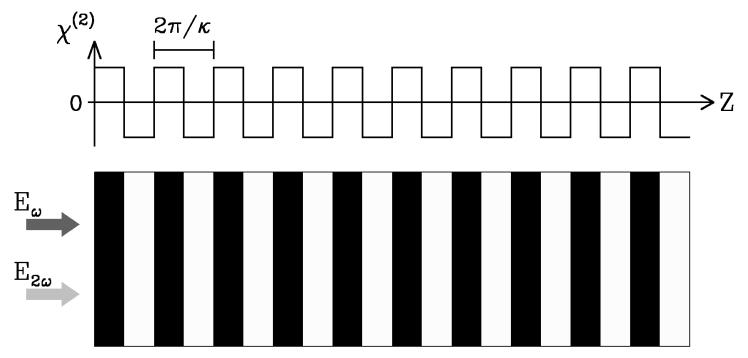

FIG. 1. Schematic presentation of a crystal with the typical square QPM modulation of the $\chi^{(2)}$ nonlinearity.
Refs. [10], we assume that the higher harmonics are of order of $1 / \kappa \ll 1$ or smaller, compared to the averages $w_{0}$ and $v_{0}$. Taking into account only the lowest order terms in the equations for the harmonics, we then derive the following relations:

$$
w_{n}=\frac{1}{m \kappa}\left(\frac{d_{(n+1) m}}{n}\right) w_{0}^{*} v_{0}, \quad v_{n}=\frac{1}{m \kappa}\left(\frac{d_{(n-1) m}}{n}\right) w_{0}^{2},
$$

Inserting the harmonics (5) into the corresponding equations for $w_{0}$ and $v_{0}$, and taking only lowest order perturbations into account, we arrive at the average equations

$$
\begin{array}{r}
i \frac{\partial w_{0}}{\partial z}+\frac{1}{2} \frac{\partial^{2} w_{0}}{\partial x^{2}}+d_{m} w_{0}^{*} v_{0}+\left(\gamma\left|w_{0}\right|^{2}+\rho\left|v_{0}\right|^{2}\right) w_{0}=0, \\
i \frac{\partial v_{0}}{\partial z}+\frac{1}{4} \frac{\partial^{2} v_{0}}{\partial x^{2}}-\tilde{\beta} v_{0}+d_{-m} w_{0}^{2}+2 \eta\left|w_{0}\right|^{2} v_{0}=0,
\end{array}
$$

where $\gamma, \rho$, and $\eta$ are all of the order of $1 / \kappa$ and given by

$$
\begin{gathered}
\gamma=\frac{1}{m \kappa} \sum_{n \neq 0} \frac{\gamma_{n}}{n}, \quad \rho=\frac{1}{m \kappa} \sum_{n \neq 0} \frac{\rho_{n}}{n}, \\
\eta=\frac{1}{m \kappa} \sum_{n \neq 0} \frac{\eta_{n}}{n}
\end{gathered}
$$

with $\gamma_{n}=d_{m(n-1)} d_{m(1-n)}, \quad \rho_{n}=d_{m(n+1)}^{*} d_{m(n+1)}, \quad$ and $\eta_{n}=d_{m(n+1)} d_{m(-n-1)}$. From Eqs. (6) follows the important result that the QPM grating introduces an effective cubic nonlinearity in the form of self- and cross-phase modulation terms. However, the self-phase modulation does not appear for the second harmonic, making the localized solutions and the system dynamics be different from the earlier analyzed case of competing nonlinearities $[9,11]$. Thus, the so-called $V$ soliton [11], where $w_{0}=0$ and $v_{0}$ is a nonlinear Schrödinger soliton, does not exist in Eqs. (6).

Let us consider the most efficient QPM of first order, $m=1$, and the square grating depicted in Fig. 1, for which the Fourier series contains odd components only. Then, the expansions for $w$ and $v$ involve only even components, and the coefficients $\gamma, \rho$, and $\eta$ become real and related

$$
\rho=\eta=-\gamma=\frac{1}{\kappa} \sum_{n \neq 0} \frac{d_{2 n-1}^{2}}{2 n} .
$$

Equations (6) therefore reduce to the following:

$$
\begin{gathered}
i \frac{\partial w_{0}}{\partial z}+\frac{1}{2} \frac{\partial^{2} w_{0}}{\partial x^{2}}-i \chi w_{0}^{*} v_{0}+\gamma\left(\left|w_{0}\right|^{2}-\left|v_{0}\right|^{2}\right) w_{0}=0 \\
i \frac{\partial v_{0}}{\partial z}+\frac{1}{4} \frac{\partial^{2} v_{0}}{\partial x^{2}}-\tilde{\beta} v_{0}+i \chi w_{0}^{2}-2 \gamma\left|w_{0}\right|^{2} v_{0}=0
\end{gathered}
$$

where both the quadratic and cubic nonlinearity coefficients are calculated in an explicit form, $\chi=2 / \pi$, $\gamma=\kappa^{-1}\left(1-8 / \pi^{2}\right)$. Note the $\pi / 2$ phase shift in front of 
the quadratic terms and the opposite signs of the cubic self- and cross-phase nonlinear terms.

We look for stationary solutions to Eqs. (7) in the form

$$
w_{0}(x, z)=\tilde{w}_{0}(x) e^{i \lambda z}, \quad v_{0}(x, z)=i \bar{v}_{0}(x) e^{2 i \lambda z} \text {, }
$$

where the real and localized profiles $\bar{w}_{0}(x)$ and $\bar{v}_{0}(x)$ are determined by the set of ordinary differential equations

$$
\begin{gathered}
\frac{1}{2} \frac{d^{2} \bar{w}_{0}}{d x^{2}}-\lambda \bar{w}_{0}+\chi \bar{w}_{0} \overline{\boldsymbol{v}}_{0}+\gamma\left(\tilde{w}_{0}^{2}-\tilde{\boldsymbol{v}}_{0}^{2}\right) \bar{w}_{0}=0, \\
\frac{1}{4} \frac{d^{2} \overline{\boldsymbol{v}}_{0}}{d x^{2}}-(\tilde{\beta}+2 \lambda) \overline{\boldsymbol{v}}_{0}+\chi \bar{w}_{0}^{2}-2 \gamma \bar{w}_{0}^{2} \overline{\boldsymbol{v}}_{0}=0,
\end{gathered}
$$

Analysis shows that localized solutions (8) exist only for positive values of the wave number $\lambda$, satisfying $\lambda>$ $\max \{0,-\tilde{\beta} / 2\}$. Note again the $\pi / 2$ phase shift appearing in the definition of the stationary solutions (8), in order for $\bar{w}_{0}(x)$ and $\bar{v}_{0}(x)$ to be real.

Localized solutions of Eqs. (9) have been found numerically for any allowed value of $\lambda$ and for the coefficients that correspond to the square grating. Figure 2 shows some of the properties of these numerical solutions for $\kappa=10$, e.g., the ratio of peak intensities vs $\lambda$ and the characteristic profiles of the solutions for $\lambda=1$, compared with the corresponding results for the zero-order approximation ( $\gamma=0$, dashed curves).

Looking at the ratio of the peak intensities shown in Fig. 2(a), the cubic correction terms are seen to have a significant effect. In the zero-order approximation $(\gamma=$ $0)$, this ratio is a constant for $\tilde{\beta}=0$, which we find numerically to be 0.6865 . However, in the QPM system with the induced Kerr effects $(\gamma \neq 0)$, this ratio tends to $\chi^{2} / 18 \gamma \lambda$ for $\lambda \gg 1$, shown as a dotted curve.

In the original variables, the stationary solutions $\bar{w}_{0}$ and $\bar{v}_{0}$ correspond to self-guided beams with rapidly oscillating intensities given by

$$
\begin{gathered}
I_{w}=|W|^{2}=\bar{w}_{0}^{2}+\frac{8}{\pi \kappa} \bar{w}_{0}^{2} \bar{v}_{0} \sum_{n=1}^{\infty} \frac{\cos (2 n \kappa z)}{4 n^{2}-1}, \\
I_{v}=|V|^{2}=\bar{v}_{0}^{2}-\frac{8}{\pi \kappa} \bar{w}_{0}^{2} \bar{v}_{0} \sum_{n=1}^{\infty} \frac{\cos (2 n \kappa z)}{4 n^{2}-1} .
\end{gathered}
$$

Since $d(z)$ is real, Eqs. (1) conserve the total power
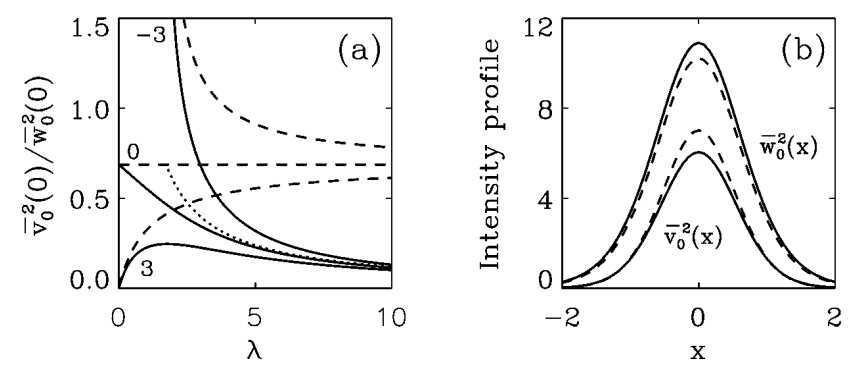

FIG. 2. Soliton families of the QPM system (7) for $\kappa=10$ (solid curves) and the zero-order approximation $(\gamma=0$, dashed curves). (a) Ratio between the peak intensity of the second harmonic, $\bar{v}_{0}^{2}(0)$, and the fundamental, $\bar{w}_{0}^{2}(0)$, vs $\lambda$. The value of $\tilde{\beta}$ is indicated at each pair of curves. The dotted curve shows the asymptotic result $\chi^{2} / 18 \gamma \lambda$. (b) Profiles $\bar{w}_{0}^{2}(x)$ and $\bar{v}_{0}^{2}(x)$ for $\tilde{\beta}=0$ and $\lambda=1$.

$$
P=\int_{-\infty}^{\infty}\left(I_{w}+I_{v}\right) d x
$$

which is also equal to the average power, since $I_{w}+$ $I_{v}=\bar{w}_{0}^{2}+\bar{v}_{0}^{2}$. The dependence of the total power on the soliton propagation constant $\lambda$ is shown in Fig. 3 for $\kappa=10$.

For negative $\tilde{\beta}$ there is a power threshold for the existence of solitons, which occurs close to the cutoff at $\lambda=\max \{0,-\tilde{\beta} / 2\}$. The induced Kerr effects are seen to increase this threshold power. However, for positive $\tilde{\beta}$ the Kerr effects decrease the power required for generating a solitary wave with a certain propagation constant $\lambda$. Stability analysis of the zero-order equations $(\gamma=0)$ has been developed in Ref. [12]. We except that similar results would apply when the induced Kerr effects are taken into account, namely that the solitary waves are stable for $d P / d \lambda>0$, and unstable for $d P / d \lambda<0$.

In order to test our asymptotic results, we use a QPM soliton as the initial condition in Eqs. (1), which we solve numerically for the square grating shown in Fig. 1. The results for $\tilde{\beta}=0$ and $\kappa=10$ are plotted in Fig. 4 and show clearly that the soliton propagates undistorted along $z$, oscillating with the period $\pi / \kappa$.

We have made a series of such numerical experiments for $\tilde{\beta}=0$, in which we propagate the QPM solitons, record their peak intensities in a while number of periods $\pi / \kappa$ after steady state is reached, and calculate the average, maximum, and minimum values in a period. The results are summarized in Fig. 5. The measured ratio of the average peak intensities of the excited solitons, shown in Fig. 5(a), deviates clearly from the prediction of the zero-order approximation $(\gamma=0)$, but is in excellent agreement with the theory that takes into account the induced Kerr effects, even for $\kappa=4$. Also the measured maximum and minimum values, shown in Fig. 5(b), are in perfect agreement with our theory. It is important to note that $\kappa=\beta=4$ corresponds to $l_{d} / l_{c}=2 / \pi$, and thus our averaged equations seem to be applicable even

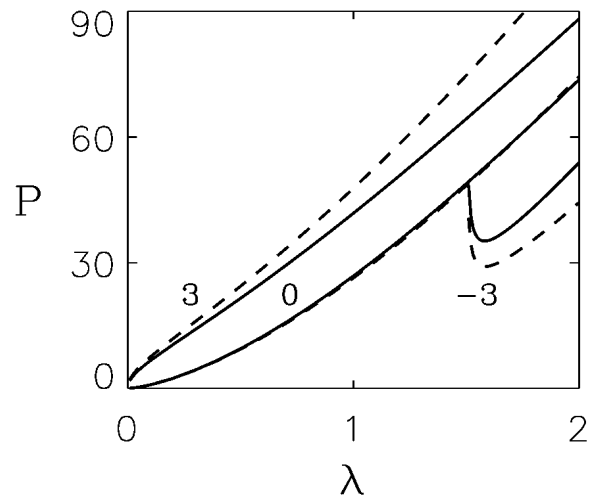

FIG. 3. Power vs propagation constant $\lambda$ for the soliton families of the QPM system (7) for $\kappa=10$ (solid curves) and the zero-order approximation $(\gamma=0$, dashed curves). The value of $\tilde{\beta}$ is indicated at each pair of curves. 

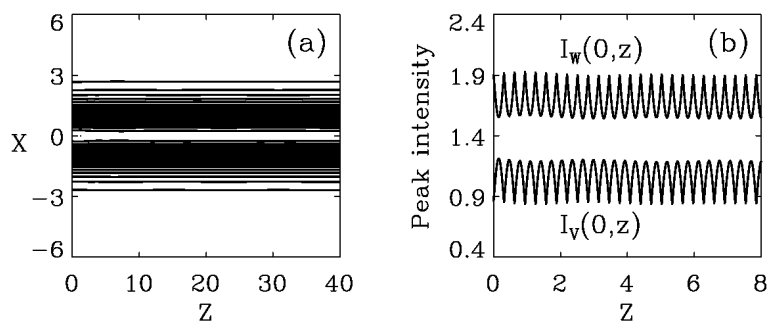

FIG. 4. Excitation of a QPM soliton with $\tilde{\beta}=0, \kappa=10$, and $\lambda=0.4$. (a) Intensity of the fundamental, $I_{w}(x, z)$, sampled at $z=n 4 \pi / \kappa$, where $n$ is an integer. Contour plot with ten equidistant levels between 0.1 and 1.92. (b) Peak intensities, $I_{w}(0, z)$ and $I_{v}(0, z)$, of the two harmonics.

when the diffraction length and the coherence length are of the same order, $l_{d} \sim l_{c}$.

We note that the average QPM soliton in quadratic media predicted in this Letter can be regarded as a spatial analog of the guided-center soliton [13], known from the theory of pulse propagation in nonlinear optical fibers with periodic amplification and dispersion management [14]. However, unlike the guided-center soliton, the periodic modulation of the quadratic nonlinearity does not alter the existing nonlinearity, it induces effective higher order nonlinearities, which we have shown can significantly modify the solitary waves.

In conclusion, we have shown that the dynamical equations describing cw beams in QPM quadratic media include periodicity-induced Kerr effects, such as self- and cross-phase modulation. These effective equations apply to the case when the diffraction length is on the order of or larger than the coherence length, $l_{d} \geq l_{c}$. We have numerically found rapidly oscillating, spatial solitary waves in the physically relevant regime of the QPM domain length and the phase mismatch. Structure and stability of these spatial QPM solitons have been investigated in the framework of the average dynamical equations, and also
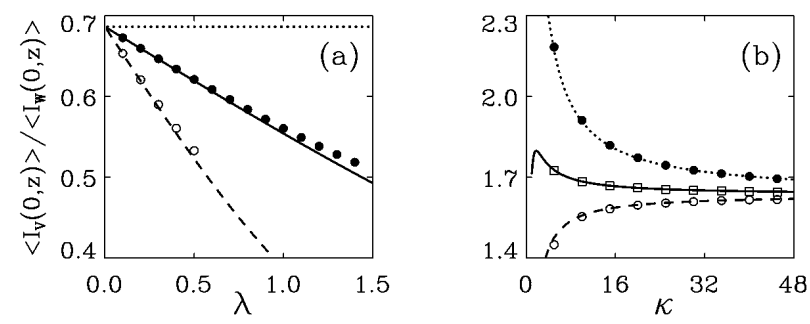

FIG. 5. Theoretical and measured (circles, dots, and squares) characteristics of the QPM solitons for $\tilde{\beta}=0$. (a) Ratio of the average peak intensities vs $\lambda$ for $\kappa=4$ (dashed curve, circles) and $\kappa=10$ (solid curve, dots). The dotted line corresponds to $\gamma=0$. (b) Mean (solid curve, squares), maximum (dotted curve, dots), and minimum (dashed curve, circles) values in a period of the fundamental peak intensity $I_{w}(0, z)$ vs $\kappa$ for $\lambda=0.4$. verified by direct numerical simulations. We believe our results reveal an important physical mechanism, by which QPM can lead, at a fixed propagation distance, to a reduction of the power required for supporting self-trapping in quadratic media.

The authors acknowledge A.V. Buryak for useful suggestions. Yu. K. is indebted to Y. Kodama for clarifying discussions of the Lie transform method, and to Professor A. Hasegawa for warm hospitality at the Osaka University.

[1] Yu. N. Karamzin and A. P. Sukhorukov, Sov. Phys. JETP 42, 842 (1976); M. J. Werner and P. D. Drummond, J. Opt. Soc. Am. B 10, 2390 (1993); A. V. Buryak and Yu. S. Kivshar, Opt. Lett. 19, 1612 (1994); Phys. Lett. A 197, 407 (1995); L. Torner, C. R. Menyuk, and G. I. Stegeman, Opt. Lett. 19, 1615 (1994); J. Opt. Soc. Am. B 12, 889 (1995); L. Bergé, V. K. Mezentsev, J. J. Rasmussen, and J. Wyller, Phys. Rev. A 52, R28 (1995); L. Torner, D. Mazilu, and D. Mihalache, Phys. Rev. Lett. 77, 2455 (1996).

[2] W.E. Torruellas, Z. Wang, D. J. Hagan, E. W. Van Stryland, G. I. Stegeman, L. Torner, and C. R. Menyuk, Phys. Rev. Lett. 74, 5036 (1995); R. Schiek, Y. Beak, and G. I. Stegeman, Phys. Rev. E 53, 1138 (1996).

[3] M. M. Fejer, G. A. Magel, D. H. Jundt, and R. L. Byer, IEEE J. Quantum Electron. 28, 2631 (1992).

[4] J. A. Armstrong, N. Bloembergen, J. Ducuing, and P. S. Pershan, Phys. Rev. 127, 1918 (1962); P. A. Franken and J. F. Ward, Rev. Mod. Phys. 35, 23 (1963).

[5] E. J. Lim, M. M. Fejer, and R. L. Byer, Electron. Lett. 25, 174 (1989).

[6] K. Mizuuchi, K. Yamamoto, and T. Taniuchi, Appl. Phys. Lett. 58, 2732 (1991).

[7] T. Fujimura, T. Suhara, and H. Nishihara, Electron. Lett. 27, 1207 (1991).

[8] C. R. Menyuk, R. Schiek, and L. Torner, J. Opt. Soc. Am. B 11, 2434 (1994).

[9] O. Bang, J. Opt. Soc. Am. B 14, 51 (1997).

[10] Yu. S. Kivshar, N. Grønbech-Jensen, and R. Parmentier, Phys. Rev. E 49, 4542 (1994); Yu. S. Kivshar and K. H. Spatschek, Chaos Solitons Fractals 5, 2551 (1995).

[11] A. V. Buryak, Yu. S. Kivshar, and S. Trillo, Opt. Lett. 20, 1961 (1995); see also Opt. Commun. 122, 200 (1996); M. A. Karpierz, Opt. Lett. 20, 1677 (1995).

[12] D. E. Pelinovsky, A. V. Buryak, and Yu. S. Kivshar, Phys. Rev. Lett. 75, 591 (1995).

[13] A. Hasegawa and Y. Kodama, Phys. Rev. Lett. 66, 161 (1991); Opt. Lett. 16, 1385 (1991).

[14] To derive an averaged equation for the guided-center soliton, the powerful Lie transform method was employed in Ref. [13] [see also A. Hasegawa and Y. Kodama, Solitons in Optical Communications (Oxford University Press, Oxford, 1995), Chap. 7, pp. 117-122, for more details] which, as can be shown, would give the result equivalent to our Eq. (6). 